\title{
Pericyclic Cascade toward Isochromenes: Application to the Synthesis of Alkaloid Benzosimuline
}

\author{
Martín J. Riveira,* Agustina La-Venia, and Mirta P. Mischne* \\ Instituto de Química Rosario, Facultad de Ciencias Bioquímicas y Farmacéuticas, Universidad Nacional de Rosario-CONICET, \\ Suipacha 531, S2002LRK Rosario, Argentina
}

\section{Supporting Information}

ABSTRACT: The synthesis of biologically active alkaloid benzosimuline, isolated from the shrub Zanthoxylum simulans, is reported. Key transformation involves an oxa- $6 \pi$ electrocyclic ringopening/hetero-Diels-Alder pericyclic cascade. Although the last aromatization step proved to be cumbersome, this work unfolds a unique route to access interesting molecules from simple precursors

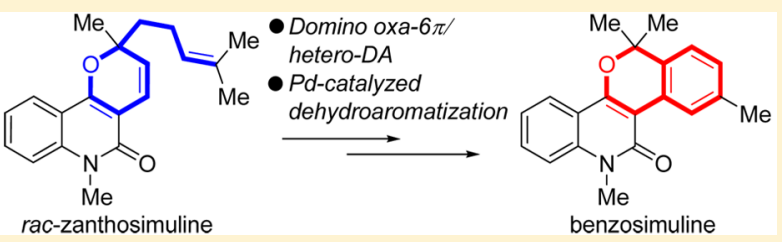

rac-zanthosimuline
7 rees and shrubs of the genus Zanthoxylum (family Lutaceae) have been the source of several unique alkaloids with varied practical pharmacological activities. ${ }^{1}$ Zanthosimuline (1), a monoterpenoid pyrano[3,2-c] quinoline, was isolated from the root bark of Taiwanese Z. simulans, from which Sichuan pepper derives, and showed to be cytotoxic against several cancer cell lines (Scheme 1). ${ }^{2}$ Related

Scheme 1. Structures of Zanthosimuline (1), Benzosimuline (2), and Possible Biosynthetic Route Involving a 3Geranylquinoline Precursor 3

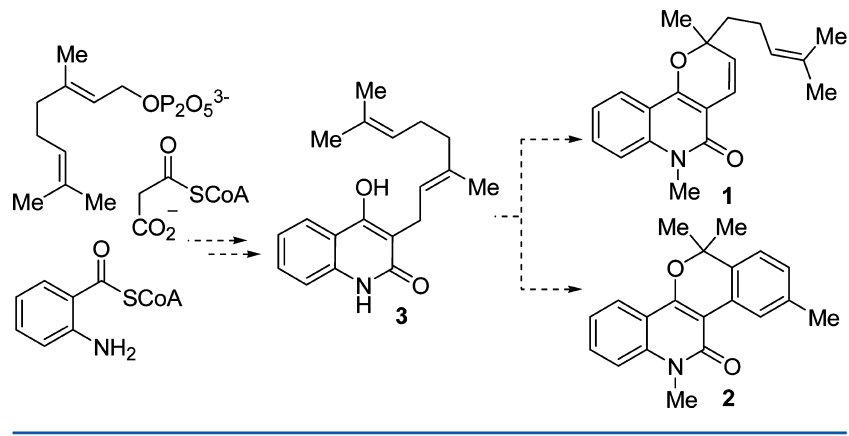

chromenoquinoline alkaloid benzosimuline (2), on the other hand, was also found in Z. simulans and exhibited cytotoxic as well as antiplatelet aggregation activity. ${ }^{3,4}$ By analogy to the chemistry of cannabinoids, ${ }^{5}$ these two alkaloids $\mathbf{1}$ and $\mathbf{2}$ related to anthranilic acid can be proposed to originate from a common synthetic intermediate, a 3-geranylquinoline motif of type 3, via different chemical transformations, probably both enzyme-catalyzed and not (Scheme 1).

As far as their syntheses are concerned, while racemic $\mathbf{1}$ had already been prepared even before the alkaloid was isolated from the natural source; ${ }^{6}$ to date and to our knowledge, there has been no synthetic approach toward natural product 2, which can only be found in 20-fold less amount than $\mathbf{1}$ in the plant. Whereas the synthesis of zanthosimuline (1) relies on the well-known domino Knoevenagel/oxa- $6 \pi$-electrocyclization process that takes place upon combination of suitable 1,3dicarbonyl substrates and $\alpha, \beta$-unsaturated aldehydes, ${ }^{7}$ the preparation of $\mathbf{2}$ would require the same substrates to engage in a different Knoevenagel/hetero-Diels-Alder cycloaddition cascade (Scheme 2). Interestingly, although this last domino sequence has been extensively studied by Tietze and others, ${ }^{8}$ it has only been observed in rare cases when using $\alpha, \beta$ unsaturated aldehydes. ${ }^{9}$ Indeed, examples are only limited to naphthoquinone substrates and yields of the desired adducts do not exceed $20 \%$.

Notwithstanding this scenario and having in mind the sensitivity of $\alpha$-pyrans toward ring opening, ${ }^{10}$ we envisaged that the core structure of $\mathbf{2}$ could also arise from $\mathbf{1}$ via a retro oxa$6 \pi$-electrocyclization/intramolecular hetero-Diels-Alder cycloaddition sequence (Scheme 2). ${ }^{11}$ A final double dehydrogenation step would then install the aromatic nucleus present in $\mathbf{2}$. Herein we wish to report the successful realization of such an approach resulting in the first synthesis of alkaloid benzosimuline.

We initiated our studies employing $2 H$-pyran model substrate 6, considering it can be prepared in almost quantitative yield from cheap and readily available substrates dimedone (4) and citral (5) using our previously reported ethylenediammonium diacetate (EDDA)-catalyzed solvent free condensation protocol (Scheme 3). ${ }^{7 \mathrm{a}}$ Since heating this substrate 6 either neat up to $170{ }^{\circ} \mathrm{C}$ or in solution up to 120 ${ }^{\circ} \mathrm{C}$ only led to traces of desired cycloadducts 9 , we considered the pericyclic cascade to be accessible through the use of acids. ${ }^{12}$ Mineral acids were screened first and found to produce extensive decomposition of substrate 6 with no trace of desired tetrahydrocannabinol (THC) analogues 9. Only in the case of using concentrated sulfuric acid could we isolate a small amount of unwanted xanthenone derivative 8 (Scheme 3 ). This

Received: May 27, 2016

Published: August 3, 2016 
Scheme 2. Domino Knoevenagel/oxa-6 $\pi$ Electrocyclization vs Knoevenagel/Hetero-Diels-Alder Cascade toward Different Pyran Scaffolds

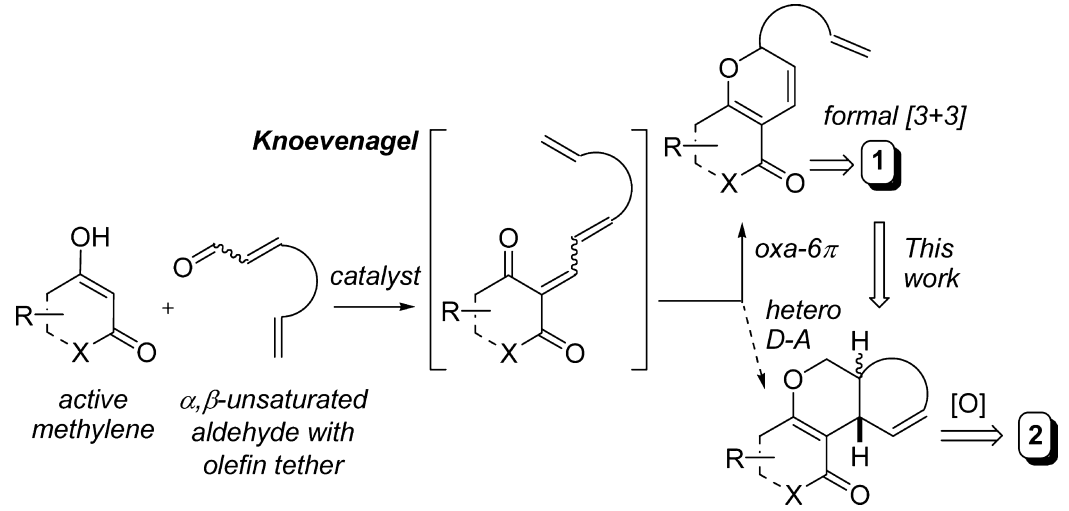

Scheme 3. Acid-Promoted Transformations of Model $2 \mathrm{H}$ Pyran Substrate 6

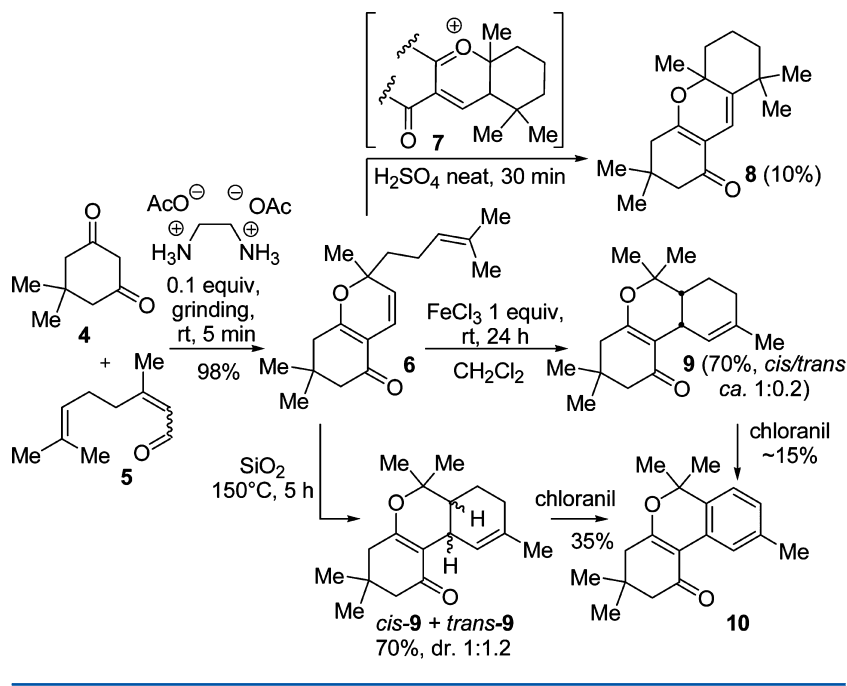

product would result from intermediate 7 formed via protonation of trisubstituted pendant olefin moiety followed by attack from the electron-rich pyran nucleus. On the other hand, it was found that several Lewis acids such as ferric chloride or Brønsted acids such as camphorsulfonic acid indeed promoted the desired pericyclic cascade at room temperature, affording tricyclic product 9 in low to moderate yields (30-
$70 \%$, see Table $\mathrm{S} 1$ in the Supporting Information for full screening data). In these cases, product 9 consisted of a mixture of cis-9 and hard to separate small amounts of the trans isomer (diastereomeric ratio ca. 1:0.2). The cis/trans ratio was calculated by integration of the ${ }^{1} \mathrm{H}$ NMR signals of the bisallylic hydrogens at 3.17 and $2.83 \mathrm{ppm}$, respectively, provided the relative configuration of these isomers was unambiguously determined by NOE experiments and by comparison with literature data on related tetrahydroisochromene derivatives. ${ }^{9 a, 11 a}$ Remarkably, although the use of higher temperatures indeed led to changes in diastereoselectivity, shifting product distribution toward the trans isomer, this was always accompanied by considerable decomposition generating complex mixtures of compounds 9 and inseparable unidentified impurities. Quite interestingly, when substrate $\mathbf{6}$ was adsorbed on silica and heated to $150{ }^{\circ} \mathrm{C}$, based on a protocol devised by Garcia et al. in the course of their studies on cannabinoid analogues, ${ }^{11 a}$ a 1:1.2 mixture of cis and trans products 9 in $70 \%$ overall yield was obtained after chromatography purification. Raising the temperature to $170{ }^{\circ} \mathrm{C}$ brought about no change in stereoselectivity and decreased yields were also obtained.

A possible mechanistic scenario for the formation of compounds 9 is depicted in Scheme 4. After an oxa-6 $\pi$ electrocyclic ring opening from 6, an intramolecular heteroDiels-Alder cycloaddition in thus formed dienone intermediate $\gamma, \delta$-Z-11 could lead to isochromene derivatives 9. Although $\gamma, \delta$ $E-11$ may as well be formed via the same pericyclic process that gives rise to $\gamma, \delta$-Z-11, it cannot lead to products 9 and may

Scheme 4. Domino Cascade Process for the Synthesis of Tetrahydroisochromenes 9

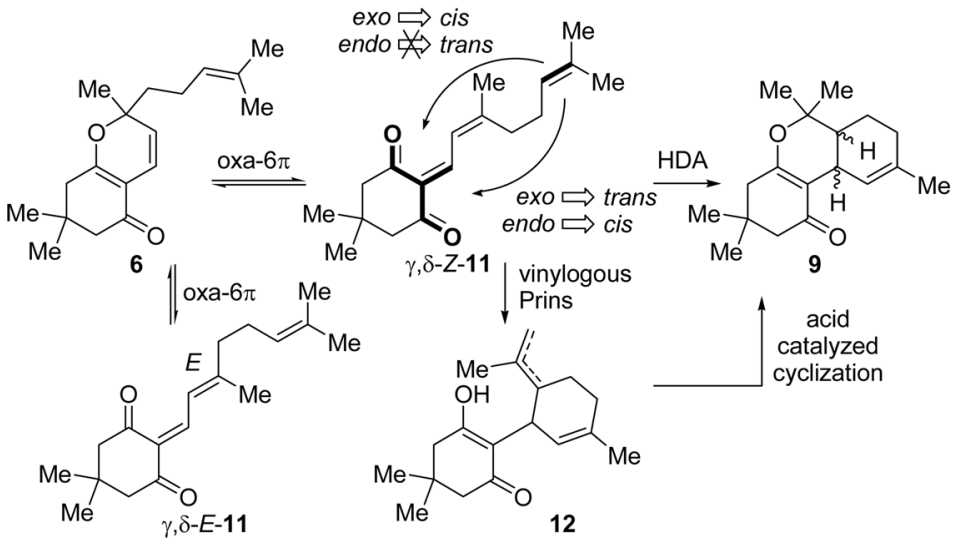

7978

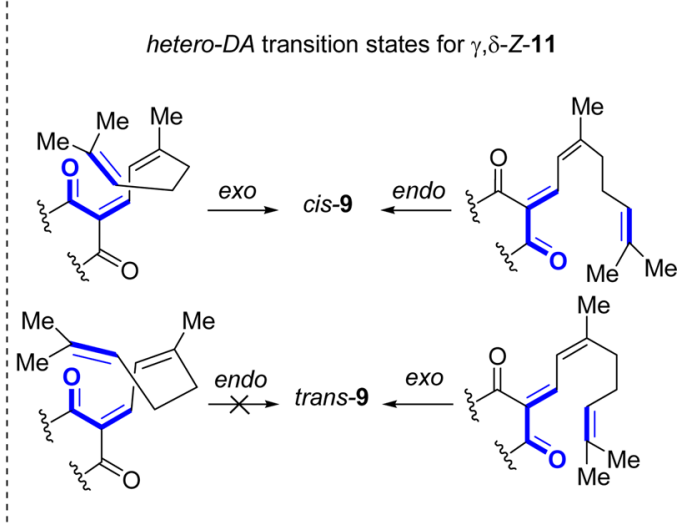


equilibrate back to starting material 6 . The hetero-Diels-Alder reaction could in principle proceed via four cyclization modes, two endo and two exo, depending on the configuration of the heterodiene and the orientation of the olefin tether. According to Tietze's studies on tandem Knoevenagel/hetero-Diels-Alder reactions, one of these modes leading to a trans-annulated product (endo $\rightarrow$ trans) can be excluded for geometrical reasons (severe angle strain). ${ }^{8,13}$ Taking this into account, and the fact that products 9 exhibited no cis-trans isomerization upon heating or by prolonging reaction times, the "exo to trans" transition structure must be higher in energy than the remaining modes of cycloaddition to account for the observed stereoselectivity shift at high temperatures. Alternatively, the construction of both new cycles in products 9 could proceed in a stepwise fashion. An initial vinylogous Prins-type reaction would lead to intermediates of type $12,{ }^{14}$ which after acid treatment could furnish bicyclized products $9 .{ }^{15}$

To our surprise, we found that these mixtures of compounds 9 were readily dehydrogenated over time into desired benzosimuline isochromene analogue 10 (Scheme 3). A literature search revealed that this chemical behavior had already been evidenced for related tetrahydrobenzochromene (-)- $\Delta^{9}-3,4-$ trans-THC, the main psychoactive constituent of cannabis, which is slowly converted to cannabinol. ${ }^{5 \mathrm{~d}, 16}$ Since air-oxidation was low-yielding, standard dehydrogenation reagents including sulfur, palladium on carbon, manganese dioxide, and high-potential quinones were then assayed to provide desired 10. Among these, chloranil was found to be the reagent of choice affording isochromene $\mathbf{1 0}$ yet in a modest $35 \%$ yield. Interestingly, the cis-9 isomer showed little, if none, reactivity and could be almost quantitatively recovered from reaction crude mixtures pointing to a strong stereoelectronic effect. Indeed, once again, marihuana scientific literature revealed the same factor affected the dehydrogenation of $\Delta^{9}$. 3,4-cis-THC, which remains unchanged for up to $20 \mathrm{~h}$ of heating with chloranil in boiling benzene. ${ }^{17}$ Consistent with these observations, the chloranil dehydrogenation of a mixture of diastereoisomers 9 obtained using $\mathrm{FeCl}_{3}$ as Lewis acid (lower trans isomer content) led to poorer oxidation yields (Scheme 3). It should also be noted that the same stereoelectronic effect on the oxidation of 9 was observed when evaluating the other standard reagents such as $\mathrm{Pd} / \mathrm{C}$ or $\mathrm{MnO}_{2}$.

Having established a route toward isochromene frameworks of type 10, at this point, we turned our attention to the synthesis of natural product 2 . To this end, rac-zanthosimuline (1) was prepared in $81 \%$ yield from citral (5) and 4-hydroxy-1methyl-2(1H)-quinolone $(13)$ as previously reported by our group (Scheme 5). ${ }^{7 \text { a }}$ Curiously, when this alkaloid $\mathbf{1}$ was adsorbed on silica gel and this mixture was heated to $150{ }^{\circ} \mathrm{C}$, cyclized products $\mathbf{1 4}$ were fortunately found albeit in lower yields than that obtained using substrate 6 and accompanied by a predominant cis-isomer content (46\% yield, dr. cis/trans: 1:0.6). Apart from desired isochromene derivatives 14, regioisomers 15 (approximately 15\% yield, dr cis/trans: 1:1.7) could also be found in the reaction mixtures, although they could not be obtained in pure form (see Supporting Information). In addition, compounds 14 turned out to be somewhat unstable, and therefore oxidation trials were preferably carried out with fresh material. When this mixture of precursors 14 was subjected to dehydrogenation conditions using chloranil, natural product benzosimuline (2) could indeed be obtained albeit in low yield $(<20 \%)$ and inseparable from an unidentified side product. After considerable
Scheme 5. Synthesis of Alkaloid Benzosimuline (2)

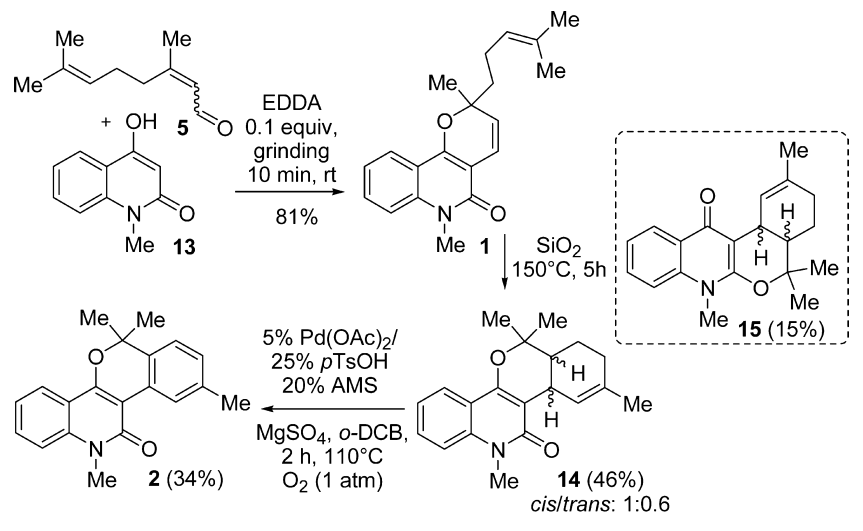

experimentation, pure 2 could be isolated as a colorless solid yet in a modest $20 \%$ yield when a mixture of compounds 14 and $10 \% \mathrm{Pd} / \mathrm{C}(20 \mathrm{~mol} \% \mathrm{Pd})$ in toluene was heated at reflux for $48 \mathrm{~h}$. Although the low trans-isomer content in $\mathbf{1 4}$ could account for the low yield of $\mathbf{2}$ formation, in these cases only $50 \%$ of original cis-14 could be recovered unaltered from reaction mixtures. An overview of marihuana chemistry shows that low yields of THC and THC analogues dehydrogenations (yields lower than 20\%) have been typical with reagents such as $\mathrm{S}$, Se or Pd/C. ${ }^{15,18}$ Dehydrogenation reactions, and in particular dehydroaromatization processes, are fundamental transformations and of increasing value both in industry and academia. ${ }^{19}$ In this regard, $\mathrm{C}-\mathrm{H}$ activation by palladium catalysis has received considerable attention these last decades. $^{20}$ Concerned about the low yield of $\mathbf{2}$ obtained upon dehydrogenation of $\mathbf{1 4}$ under heterogeneous conditions, we set out to test some modern oxidation protocols. After some frustrated attempts, a slightly modified procedure of that recently published by Stahl's group fortunately allowed for a slight increase in natural product 2 formation. ${ }^{20 a}$ Thus, treatment of compounds 14 with $\mathrm{Pd}(\mathrm{OAc})_{2}$ in combination with $p$-toluenesulfonic acid $(p \mathrm{TsOH})$ and sodium anthraquinone-2-sulfonate (AMS) in 1,2-dichlorobenzene at $110^{\circ} \mathrm{C}$ for $2 \mathrm{~h}$ using oxygen as terminal oxidant ( $1 \mathrm{~atm})$ led to the synthesis of $\mathbf{2}$ in $34 \%$ yield. Together with desired natural product, a small amount of unreacted cis-14 (12\%) could also be found in the complex crude mixture.

Having achieved the first synthesis of alkaloid benzosimuline (2), in order to test the scope of the process, we next evaluated the extension of this methodology to the synthesis of $\Delta^{9}$ tetrahydrocannabiorcol (18) and aromatized derivative cannabiorcol (19), both cannabinoids identified in extracts of Brazilian marihuana (Scheme 6A). ${ }^{21}$ To this end, the requisite precursor cannabichromene- $\mathrm{C}_{1}(17)$ was prepared from orcinol (16) and citral (5) as reported by Lee and co-workers. ${ }^{22,23}$ Accordingly, benzopyran 17 was adsorbed on silica gel, and this mixture was heated to $150{ }^{\circ} \mathrm{C}$. After $7 \mathrm{~h},{ }^{1} \mathrm{H}$ NMR monitoring of the crude mixture indicated complete consumption of starting pyran substrate 17 , and after chromatography purification, only three products could be isolated in low yields, 20, 21 and 22; these last two as an inseparable mixture. Both 20 and 21 are biologically active natural products rhanuadujuanine $\mathrm{B}$ and $\mathrm{A}$ isolated from the Chinese medicinal plant Rhododendron anthopogonoides. ${ }^{24}$ The structure of compound 22, on the other hand, corresponds to the cannabinoid class iso-THC's. The constitution of the only isolable products 20-22 suggests that these conditions would 
Scheme 6. Thermal Rearrangement of Cannabichromene- $C_{1}$ (17) Adsorbed on Silica

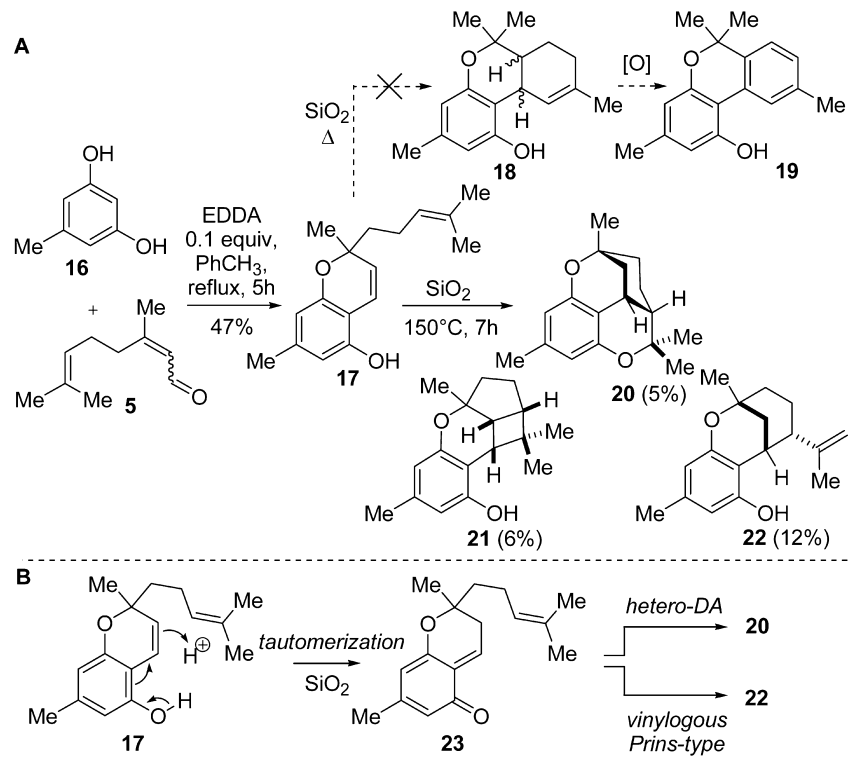

not be suitable to promote the ring opening of the pyran core in chromenol substrates. While rhanuadujuanine B (21) would be the result of a Gassman-like cationic $[2+2]$ cycloaddition, ${ }^{24,25}$ products $\mathbf{2 0}$ and $\mathbf{2 2}$ would result, respectively, from hetero-Diels-Alder and vinylogous Prins-type reactions of $o$-quinone methide intermediate $\mathbf{2 3}$ formed via tautomerization of substrate 17 (Scheme $6 \mathrm{~B}) .^{26}$

In summary, a novel pericyclic cascade for the synthesis of the isochromene natural motif has been explored, and its application in combination with a Pd-catalyzed dehydroaromatization reaction allowed for the first synthesis of alkaloid benzosimuline (2). Initial attempts to extend this methodology to the preparation of known cannabinoids foundered but gave interesting insights into the chemical reactivity of chromenes.

\section{EXPERIMENTAL SECTION}

General Information. Precursors zanthosimuline (1) and $\mathbf{6}$ have been previously prepared by our group using a solvent-free condensation protocol. ${ }^{\text {7a }}$ Precursor cannabichromene- $\mathrm{C}_{1}(17)$ has been prepared according to a literature procedure. ${ }^{22}$ All other reagents were purchased from commercial suppliers and used without further purification. Thin-layer chromatography was performed on silica gel 60 $\mathrm{F}_{254}$ precoated aluminum sheets. The products were purified by column chromatography using silica gel 60 (230-400 mesh). Nuclear magnetic resonance spectra were acquired at $300 \mathrm{MHz}$ for ${ }^{1} \mathrm{H}$ and 75 $\mathrm{MHz}$ for ${ }^{13} \mathrm{C}$ using $\mathrm{CDCl}_{3}$ as solvent. Chemical shifts are reported in ppm and coupling constants $(J)$ are reported in hertz $(\mathrm{Hz})$. IR spectra were obtained using an FT-IR spectrometer, and only partial spectral data are listed. High-resolution mass spectra (HRMS) were recorded with a Q-TOF mass spectrometer equipped with an ESI source (detection of the ions was performed in electrospray ionization, positive ion mode).

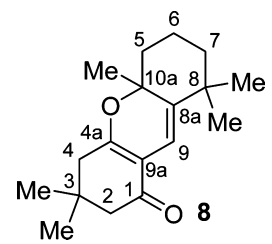

Sulfuric Acid-Promoted Rearrangement of Substrate 6 to 8 . In a flask containing substrate $6(40 \mathrm{mg}, 0.14 \mathrm{mmol})$ was added $98 \%$ sulfuric acid $(0.5 \mathrm{~mL})$ at $0{ }^{\circ} \mathrm{C}$. The mixture was stirred for $30 \mathrm{~min}$ at room temperature, and then brine $(40 \mathrm{~mL})$ and ethyl ether $(40 \mathrm{~mL})$ were added. The organic layer was then washed twice with saturated $\mathrm{NaHCO}_{3}$ solution $(30 \mathrm{~mL})$. The residue was purified by flash column chromatography on silica gel (eluent hexanes/ethyl acetate) to afford 8 in $10 \%$ yield $(4 \mathrm{mg}, 0.014 \mathrm{mmol})$.

Compound 8. Colorless liquid. IR (film) $\left(\mathrm{cm}^{-1}\right): 2959,2934,2870$, $1715,1626,1601,1462,1385 .{ }^{1} \mathrm{H}$ NMR $\left(\mathrm{CDCl}_{3}, 300 \mathrm{MHz}\right): \delta 6.32(\mathrm{~s}$, $1 \mathrm{H}, 9-\mathrm{H}), 2.30(\mathrm{dAB}, J=16.1 \mathrm{~Hz}, 1 \mathrm{H}, 2-\mathrm{H}), 2.26$ (bs, $2 \mathrm{H}, 4-\mathrm{H}), 2.20$ $(\mathrm{dAB}, J=16.3 \mathrm{~Hz}, 1 \mathrm{H}, 2-\mathrm{H}), 2.03\left(\mathrm{dm}, J=12.6 \mathrm{~Hz}, 1 \mathrm{H}, 5-\mathrm{H}_{\mathrm{eq}}\right), 1.81$ $\left(\mathrm{td}, J=12.5 \mathrm{~Hz}, J=5.0 \mathrm{~Hz}, 1 \mathrm{H}, 5-\mathrm{H}_{\mathrm{ax}}\right), 1.73-1.52(\mathrm{~m}, 2 \mathrm{H}, 6-\mathrm{H})$, $1.50-1.35(\mathrm{~m}, 2 \mathrm{H}, 7-\mathrm{H}), 1.41\left(\mathrm{~s}, 3 \mathrm{H}, 10 \mathrm{a}-\mathrm{CH}_{3}\right), 1.21\left(\mathrm{~s}, 3 \mathrm{H}, 8-\mathrm{CH}_{3}\right)$, $1.11\left(\mathrm{~s}, 3 \mathrm{H}, 8-\mathrm{CH}_{3}\right), 1.09\left(\mathrm{~s}, 3 \mathrm{H}, 3-\mathrm{CH}_{3}\right), 1.04\left(\mathrm{~s}, 3 \mathrm{H}, 3-\mathrm{CH}_{3}\right) .{ }^{13} \mathrm{C}$ NMR ( $\left.\mathrm{CDCl}_{3}, 75 \mathrm{MHz}\right): \delta 194.9$ (C, C-1), 168.3 (C, C-4a), 139.6 (C, C-8a), 111.4 (C, C-9a), 109.8 (CH, C-9), 81.7 (C, C-10a), $50.5\left(\mathrm{CH}_{2}\right.$, $\mathrm{C}-2), 42.1\left(\mathrm{CH}_{2}, \mathrm{C}-4\right), 39.6\left(\mathrm{CH}_{2}, \mathrm{C}-5\right), 39.3\left(\mathrm{CH}_{2}, \mathrm{C}-7\right), 35.4(\mathrm{C}, \mathrm{C}-$ 8), $32.2(\mathrm{C}, \mathrm{C}-3), 30.6\left(\mathrm{CH}_{3}, \mathrm{C} 8-\mathrm{CH}_{3}\right), 30.5\left(\mathrm{CH}_{3}, \mathrm{C} 8-\mathrm{CH}_{3}\right), 29.4$ $\left(\mathrm{CH}_{3}, \mathrm{C} 3-\mathrm{CH}_{3}\right), 27.4\left(\mathrm{CH}_{3}, \mathrm{C} 3-\mathrm{CH}_{3}\right), 25.8\left(\mathrm{CH}_{3}, \mathrm{ClOa}-\mathrm{CH}_{3}\right), 18.9$ $\left(\mathrm{CH}_{2}, \mathrm{C}-6\right)$. HRMS (ESI) $\mathrm{m} / z$ calcd for $\mathrm{C}_{18} \mathrm{H}_{26} \mathrm{O}_{2} \mathrm{Na}[\mathrm{M}+\mathrm{Na}]^{+}$ 297.1825, found 297.1816.

General Procedure for the Rearrangement of Substrates 1, 6 , and 17 Adsorbed on Silica. The substrate $(1 \mathrm{mmol})$ was adsorbed on silica gel $(300 \mathrm{w} / \mathrm{w} \%, 60,0.063-0.200 \mathrm{~mm})$ and heated to $150{ }^{\circ} \mathrm{C}$ for $5 \mathrm{~h}$ in the case of substrates 1 and 6 and $7 \mathrm{~h}$ for precursor 17. TLC monitoring can be performed by taking samples and extracting the compounds with a small volume of AcOEt. After completion of the reaction, the residue was directly purified by flash column chromatography on silica gel (eluent hexanes/ethyl acetate) to afford the following products.
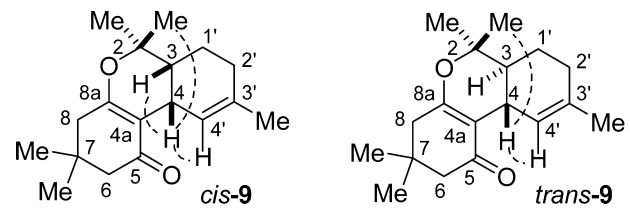

Compounds cis-9 + trans-9. Obtained from substrate 6 (1.0 mmol, $274 \mathrm{mg})$ as a pale yellow liquid in $70 \%$ yield $(0.7 \mathrm{mmol}, 192$ $\mathrm{mg}$, cis/trans ratio $=1: 1.2)$. IR $($ film $)\left(\mathrm{cm}^{-1}\right): 2957,2870,1651,1601$, 1366, 1120. ${ }^{1} \mathrm{H}$ NMR $\left(\mathrm{CDCl}_{3}, 300 \mathrm{MHz}\right)$ : cis-9 $\delta 6.13-6.03(\mathrm{~m}, 1 \mathrm{H}$, 4'-H), 3.17 (bs, $1 \mathrm{H}, 4-\mathrm{H}), 2.41-2.08$ (m, 4H, 6-H, 8-H), 1.99-1.90 $\left(\mathrm{m}, 2 \mathrm{H}, 2^{\prime}-\mathrm{H}\right), 1.89-1.75\left(\mathrm{~m}, 1 \mathrm{H}, 1^{\prime}-\mathrm{H}\right), 1.68-1.57(\mathrm{~m}, 1 \mathrm{H}, 3-\mathrm{H})$, 1.64 (bs, $\left.3 \mathrm{H}, 3^{\prime}-\mathrm{CH}_{3}\right), 1.36\left(\mathrm{~s}, 3 \mathrm{H}, 2-\mathrm{CH}_{3 \alpha}\right), 1.29-1.20\left(\mathrm{~m}, 1 \mathrm{H}, \mathrm{l}^{\prime}-\right.$ $\mathrm{H}), 1.23\left(\mathrm{~s}, 3 \mathrm{H}, 2-\mathrm{CH}_{3 \beta}\right), 1.03\left(\mathrm{~s}, 6 \mathrm{H}, 7-\mathrm{CH}_{3}, 7-\mathrm{CH}_{3}\right)$; trans $-9 \delta 6.10-$ $6.04\left(\mathrm{~m}, 1 \mathrm{H}, 4^{\prime}-\mathrm{H}\right), 2.83(\mathrm{bd}, J=10.5 \mathrm{~Hz}, 1 \mathrm{H}, 4-\mathrm{H}), 2.41-2.08(\mathrm{~m}$, $4 \mathrm{H}, 6-\mathrm{H}, 8-\mathrm{H}), 2.08-2.05\left(\mathrm{~m}, 2 \mathrm{H}, 2^{\prime}-\mathrm{H}\right), 1.89-1.75\left(\mathrm{~m}, 1 \mathrm{H}, 1^{\prime}-\mathrm{H}\right)$, $1.64\left(\mathrm{bs}, 3 \mathrm{H}, 3^{\prime}-\mathrm{CH}_{3}\right), 1.51$ (ddd, $J=12.5 \mathrm{~Hz}, J=10.5 \mathrm{~Hz}, J=1.8 \mathrm{~Hz}$, 3-H), 1.40-1.29 (m, 1H, 1'-H), $1.38\left(\mathrm{~s}, 3 \mathrm{H}, 2-\mathrm{CH}_{3 \alpha}\right), 1.07$ (s, 6H, 7$\left.\mathrm{CH}_{3}, 2-\mathrm{CH}_{3 \beta}\right), 1.03\left(\mathrm{~s}, 3 \mathrm{H}, 7-\mathrm{CH}_{3}\right) .{ }^{13} \mathrm{C} \mathrm{NMR}\left(\mathrm{CDCl}_{3}, 75 \mathrm{MHz}\right)$ : cis$9 \delta 197.8$ (C, C-5), 166.8 (C, C-8a), 133.2 (C, C-3'), 121.9 (CH, C$\left.4^{\prime}\right), 112.4$ (C, C-4a), 78.9 (C, C-2), $51.3\left(\mathrm{CH}_{2}, \mathrm{C}-6\right), 42.9\left(\mathrm{CH}_{2}, \mathrm{C}-8\right)$, 39.8 (CH, C-3), 31.7 (C, C-7), $29.7\left(\mathrm{CH}, \mathrm{C}-4 ; \mathrm{CH}_{2}, \mathrm{C}-2^{\prime}\right), 28.6$ $\left(\mathrm{CH}_{3}, \mathrm{C} 7-\mathrm{CH}_{3}\right), 27.5\left(\mathrm{CH}_{3}, \mathrm{C} 7-\mathrm{CH}_{3}\right), 25.5\left(\mathrm{CH}_{3}, 2-\mathrm{CH}_{3 \alpha}\right), 25.1$ $\left(\mathrm{CH}_{3}, 2-\mathrm{CH}_{3 \beta}\right), 23.4\left(\mathrm{CH}_{3}, \mathrm{C}^{\prime}-\mathrm{CH}_{3}\right), 20.3\left(\mathrm{CH}_{2}, \mathrm{C}-1^{\prime}\right)$; trans-9 $\delta$ 197.2 (C, C-5), 168.7 (C, C-8a), 133.0 (C, C-3'), 123.4 (CH, C-4'), 111.8 (C, C-4a), 80.5 (C, C-2), $51.3\left(\mathrm{CH}_{2}, \mathrm{C}-6\right), 45.3(\mathrm{CH}, \mathrm{C}-3), 43.0$ $\left(\mathrm{CH}_{2}, \mathrm{C}-8\right), 31.8(\mathrm{CH}, \mathrm{C}-4), 31.7$ (C, C-7), $31.1\left(\mathrm{CH}_{2}, \mathrm{C}-2^{\prime}\right), 28.8$ $\left(\mathrm{CH}_{3}, \mathrm{C} 7-\mathrm{CH}_{3}\right), 27.5\left(\mathrm{CH}_{3}, \mathrm{C} 7-\mathrm{CH}_{3}\right), 26.9\left(\mathrm{CH}_{3}, 2-\mathrm{CH}_{3 \alpha}\right), 24.4$ $\left(\mathrm{CH}_{2}, \mathrm{C}-1^{\prime}\right), 23.1\left(\mathrm{CH}_{3}, \mathrm{C}^{\prime}-\mathrm{CH}_{3}\right), 19.8\left(\mathrm{CH}_{3}, 2-\mathrm{CH}_{3 \beta}\right)$. HRMS (ESI) $\mathrm{m} / z$ calcd for $\mathrm{C}_{18} \mathrm{H}_{27} \mathrm{O}_{2}[\mathrm{M}+\mathrm{H}]^{+} 275.2006$, found 275.2004.
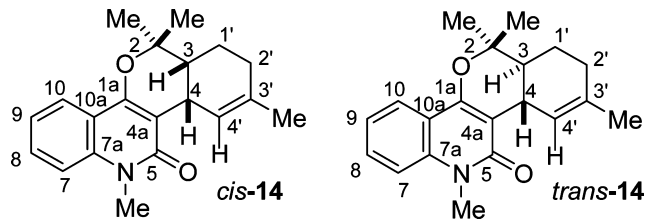

Compounds cis-14 + trans-14. Obtained from substrate 1 (1.0 $\mathrm{mmol}, 309 \mathrm{mg})$ as a pale yellow liquid in $46 \%$ yield $(0.46 \mathrm{mmol}, 142$ $\mathrm{mg}$, cis/trans ratio $=1: 0.6)$. IR (film) $\left(\mathrm{cm}^{-1}\right): 2976,2936,1634,1589$, 1504, 1391. ${ }^{1} \mathrm{H}$ NMR $\left(\mathrm{CDCl}_{3}, 300 \mathrm{MHz}\right):$ cis-14 $\delta 7.94(\mathrm{dd}, J=8.0$ $\mathrm{Hz}, J=1.3 \mathrm{~Hz}, 1 \mathrm{H}, 10-\mathrm{H}$ ), 7.50 (overlapping bt, $J=8.0 \mathrm{~Hz}, 1 \mathrm{H}, 8-\mathrm{H}$ ), 
$7.28(\mathrm{~d}, J=8.4 \mathrm{~Hz}, 1 \mathrm{H}, 7-\mathrm{H}$ ), 7.18 (overlapping bt, $J=7.7 \mathrm{~Hz}, 1 \mathrm{H}, 9$ H), $6.50-6.43\left(\mathrm{~m}, 1 \mathrm{H}, 4^{\prime}-\mathrm{H}\right), 3.68\left(\mathrm{~s}, 3 \mathrm{H}, \mathrm{N}-\mathrm{CH}_{3}\right), 3.55$ (bs, $1 \mathrm{H}, 4-$ $\mathrm{H}), 2.03-1.87\left(\mathrm{~m}, 3 \mathrm{H}, 2^{\prime}-\mathrm{H}, 2^{\prime}-\mathrm{H}, 1^{\prime}-\mathrm{H}\right), 1.87-1.75(\mathrm{~m}, 1 \mathrm{H}, 3-\mathrm{H})$, $1.69\left(\mathrm{bs}, 3 \mathrm{H}, 3^{\prime}-\mathrm{CH}_{3}\right), 1.53\left(\mathrm{~s}, 3 \mathrm{H}, 2-\mathrm{CH}_{3}\right), 1.44-1.32\left(\mathrm{~m}, 1 \mathrm{H}, \mathrm{1}^{\prime}-\mathrm{H}\right)$, $1.33\left(\mathrm{~s}, 3 \mathrm{H}, 2-\mathrm{CH}_{3}\right)$; trans-14 $\delta 7.95(\mathrm{dd}, J=7.9 \mathrm{~Hz}, J=1.2 \mathrm{~Hz}, 1 \mathrm{H}$, $10-\mathrm{H}), 7.50$ (overlapping bt, $J=8.0 \mathrm{~Hz}, 1 \mathrm{H}, 8-\mathrm{H}), 7.29(\mathrm{~d}, J=8.4 \mathrm{~Hz}$, $1 \mathrm{H}, 7-\mathrm{H}$ ), 7.18 (overlapping bt, $J=7.7 \mathrm{~Hz}, 1 \mathrm{H}, 9-\mathrm{H}), 6.50-6.43(\mathrm{~m}$, $\left.1 \mathrm{H}, 4^{\prime}-\mathrm{H}\right), 3.69\left(\mathrm{~s}, 3 \mathrm{H}, \mathrm{N}-\mathrm{CH}_{3}\right), 3.22(\mathrm{dm}, J=10.8 \mathrm{~Hz}, 1 \mathrm{H}, 4-\mathrm{H})$, 2.23-2.12 (m, 2H, 2'-H), 2.03-1.87 (m, 1H, 1'- $\mathrm{H}), 1.75-1.62(\mathrm{~m}$, $1 \mathrm{H}, 3-\mathrm{H}), 1.69$ (bs, 3H, 3'- $\left.\mathrm{CH}_{3}\right), 1.55\left(\mathrm{~s}, 3 \mathrm{H}, 2-\mathrm{CH}_{3}\right), 1.54-1.43(\mathrm{~m}$, $\left.1 \mathrm{H}, 1^{\prime}-\mathrm{H}\right), 1.16\left(\mathrm{~s}, 3 \mathrm{H}, 2-\mathrm{CH}_{3}\right) .{ }^{13} \mathrm{C} \mathrm{NMR}\left(\mathrm{CDCl}_{3}, 75 \mathrm{MHz}\right): c i s-14 \delta$ 162.9 (C, C-5), 154.1 (C, C-1a), 138.6 (C, C-7a), 134.2 (C, C-3'), $129.8(\mathrm{CH}, \mathrm{C}-8), 122.9$ (C, C-10), $121.2\left(\mathrm{CH}, \mathrm{C}-4^{\prime}\right), 121.1(\mathrm{CH}, \mathrm{C}-$ 9), 116.6 (C, C-10a), 113.41 (CH, C-7), 108.8 (C, C-4a), 78.5 (C, C2), $39.9(\mathrm{CH}, \mathrm{C}-3), 31.5(\mathrm{CH}, \mathrm{C}-4), 29.7\left(\mathrm{CH}_{2}, \mathrm{C}-2^{\prime}\right), 29.0\left(\mathrm{CH}_{3}, \mathrm{~N}-\right.$ $\left.\mathrm{CH}_{3}\right), 25.7\left(\mathrm{CH}_{3}, \mathrm{C} 2-\mathrm{CH}_{3}\right), 24.8\left(\mathrm{CH}_{3}, \mathrm{C} 2-\mathrm{CH}_{3}\right), 23.5\left(\mathrm{CH}_{3}, \mathrm{C}^{\prime}-\right.$ $\left.\mathrm{CH}_{3}\right), 20.5\left(\mathrm{CH}_{2}, \mathrm{C}-1^{\prime}\right)$; trans-14 $\delta 162.5(\mathrm{C}, \mathrm{C}-5), 155.4(\mathrm{C}, \mathrm{C}-1 \mathrm{a})$, 138.7 (C, C-7a), 133.4 (C, C-3'), 129.9 (CH, C-8), 123.0 (C, C-10), $122.6\left(\mathrm{CH}, \mathrm{C}-4^{\prime}\right), 121.1(\mathrm{CH}, \mathrm{C}-9), 116.7$ (C, C-10a), $113.44(\mathrm{CH}$, C-7), 107.7 (C, C-4a), 79.8 (C, C-2), 45.5 (CH, C-3), 33.7 (CH, C-4), $31.2\left(\mathrm{CH}_{2}, \mathrm{C}-2^{\prime}\right), 28.9\left(\mathrm{CH}_{3}, \mathrm{~N}-\mathrm{CH}_{3}\right), 27.1\left(\mathrm{CH}_{3}, \mathrm{C} 2-\mathrm{CH}_{3}\right), 24.7$ $\left(\mathrm{CH}_{2}, \mathrm{C}-1^{\prime}\right), 23.1\left(\mathrm{CH}_{3}, \mathrm{C}^{\prime}-\mathrm{CH}_{3}\right), 19.5\left(\mathrm{CH}_{3}, \mathrm{C} 2-\mathrm{CH}_{3}\right)$. HRMS (ESI) $\mathrm{m} / z$ calcd for $\mathrm{C}_{40} \mathrm{H}_{46} \mathrm{~N}_{2} \mathrm{O}_{4} \mathrm{Na}[2 \mathrm{M}+\mathrm{Na}]^{+}$641.3350, found 641.3330 .
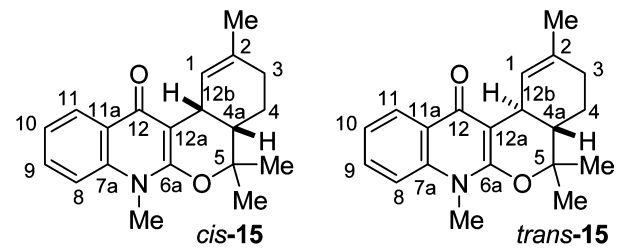

Compounds 15. Obtained from substrate $1(1.0 \mathrm{mmol}, 309 \mathrm{mg})$ as side products in approximately $15 \%$ yield $(0.15 \mathrm{mmol}, 46 \mathrm{mg}$, cis/ trans ratio $=1: 1.7)$. Only the cis isomer (cis-15) could be characterized since trans-15 is inseparable from impurities (see Supporting Information). Pale orange liquid. IR (film) $\left(\mathrm{cm}^{-1}\right)$ : 2926, 1620, 1597, 1537, 1504. ${ }^{1} \mathrm{H}$ NMR $\left(\mathrm{CDCl}_{3}, 300 \mathrm{MHz}\right)$ : cis-15 $\delta 8.42$ (dd, $J=$ $7.9 \mathrm{~Hz}, J=1.5 \mathrm{~Hz}, 1 \mathrm{H}, 11-\mathrm{H}), 7.58(\mathrm{ddd}, J=8.6 \mathrm{~Hz}, J=7.0 \mathrm{~Hz}, J=1.6$ $\mathrm{Hz}, 1 \mathrm{H}, 9-\mathrm{H}), 7.37$ (d, $J=8.3 \mathrm{~Hz}, 1 \mathrm{H}, 8-\mathrm{H}), 7.29$ (ddd, $J=8.0 \mathrm{~Hz}, J=$ $7.1 \mathrm{~Hz}, J=0.9 \mathrm{~Hz}, 1 \mathrm{H}, 10-\mathrm{H}), 6.61(\mathrm{bd}, J=3.4 \mathrm{~Hz}, 1 \mathrm{H}, 1-\mathrm{H}), 3.71(\mathrm{bs}$, $1 \mathrm{H}, 12 \mathrm{~b}-\mathrm{H}), 3.65\left(\mathrm{~s}, 3 \mathrm{H}, \mathrm{N}-\mathrm{CH}_{3}\right), 2.04-1.89(\mathrm{~m}, 3 \mathrm{H}, 3-\mathrm{H}, 3-\mathrm{H}, 4-$ $\mathrm{H}), 1.88-1.77(\mathrm{~m}, 1 \mathrm{H}, 4 \mathrm{a}-\mathrm{H}), 1.67\left(\mathrm{bs}, 3 \mathrm{H}, 2-\mathrm{CH}_{3}\right), 1.54(\mathrm{~s}, 3 \mathrm{H}, 5-$ $\left.\mathrm{CH}_{3 \alpha}\right), 1.42-1.36(\mathrm{~m}, 1 \mathrm{H}, 4-\mathrm{H}), 1.38\left(\mathrm{~s}, 3 \mathrm{H}, 5-\mathrm{CH}_{3 \beta}\right) .{ }^{13} \mathrm{C}$ NMR $\left(\mathrm{CDCl}_{3}, 75 \mathrm{MHz}\right):$ cis-15 $\delta 176.8(\mathrm{C}, \mathrm{C}-12), 154.0(\mathrm{C}, \mathrm{C}-6 \mathrm{a}), 138.8$ (C, C-7a), 133.1 (C, C-2), 131.1 (CH, C-9), 126.4 (CH, C-11), 124.7 (C, C-11a), 122.3 (CH, C-10), $121.6(\mathrm{CH}, \mathrm{C}-1), 114$. One (CH, C-8), 101.8 (C, C-12a), 81.9 (C, C-5), 39.7 (CH, C-4a), 30.8 (CH, C-12b), $30.2\left(\mathrm{CH}_{3}, \mathrm{~N}-\mathrm{CH}_{3}\right), 29.5\left(\mathrm{CH}_{2}, \mathrm{C}-3\right), 25.6\left(\mathrm{CH}_{3}, \mathrm{C} 5-\mathrm{CH}_{3 \alpha}\right), 25.2$ $\left(\mathrm{CH}_{3}, \mathrm{C} 2-\mathrm{CH}_{3 \beta}\right), 23.4\left(\mathrm{CH}_{3}, \mathrm{C} 2-\mathrm{CH}_{3}\right), 20.5\left(\mathrm{CH}_{2}, \mathrm{C}-4\right)$. HRMS (ESI) $m / z$ calcd for $\mathrm{C}_{20} \mathrm{H}_{24} \mathrm{NO}_{2}[\mathrm{M}+\mathrm{H}]^{+} 310.1802$, found 310.1813 .

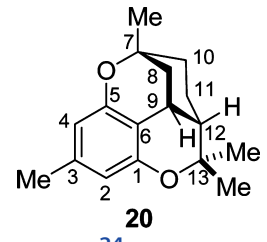

Ranhuadujuanine B (20). ${ }^{24}$ Obtained from substrate 17 (1.0 $\mathrm{mmol}, 258 \mathrm{mg})$ as a pale yellow solid in $5 \%$ yield $(0.05 \mathrm{mmol}, 13 \mathrm{mg})$. Mp 135.0-137.0 ${ }^{\circ} \mathrm{C} .{ }^{1} \mathrm{H}$ NMR $\left(\mathrm{CDCl}_{3}, 300 \mathrm{MHz}\right): \delta 6.31$ (bs, $1 \mathrm{H}, 4-$ $\mathrm{H}), 6.26(\mathrm{bs}, 1 \mathrm{H}, 2-\mathrm{H}), 2.82(\mathrm{bs}, 1 \mathrm{H}, 9-\mathrm{H}), 2.25\left(\mathrm{~s}, 3 \mathrm{H}, 3-\mathrm{CH}_{3}\right), 2.21$ (ddd, $J=13.1 \mathrm{~Hz}, J=4.6 \mathrm{~Hz}, J=3.3 \mathrm{~Hz}, 1 \mathrm{H}, 8-\mathrm{H}), 2.01(\mathrm{ddd}, J=11.6$ $\mathrm{Hz}, J=5.3 \mathrm{~Hz}, J=2.7 \mathrm{~Hz}, 1 \mathrm{H}, 12-\mathrm{H}), 1.82(\mathrm{dd}, J=13.3 \mathrm{~Hz}, J=1.6$ $\mathrm{Hz}, 1 \mathrm{H}, 8-\mathrm{H}), 1.75(\mathrm{dm}, J=14.5 \mathrm{~Hz}, 1 \mathrm{H}, 10-\mathrm{H}), 1.51(\mathrm{~s}, 3 \mathrm{H}, 13-$ $\mathrm{CH}_{3 \beta}$ ), 1.41 (ddd, $J=14.5 \mathrm{~Hz}, J=13.2 \mathrm{~Hz}, J=6.7 \mathrm{~Hz}, 1 \mathrm{H}, 10-\mathrm{H}$ ), $1.37\left(\mathrm{~s}, 3 \mathrm{H}, 7-\mathrm{CH}_{3}\right), 1.23(\mathrm{bdt}, J=13.7 \mathrm{~Hz}, J=5.8 \mathrm{~Hz}, 1 \mathrm{H}, 11-\mathrm{H})$, $1.01\left(\mathrm{~s}, 3 \mathrm{H}, 13-\mathrm{CH}_{3 \alpha}\right), 0.70-0.52(\mathrm{~m}, 1 \mathrm{H}, 11-\mathrm{H}) .{ }^{13} \mathrm{C} \mathrm{NMR}\left(\mathrm{CDCl}_{3}\right.$, $75 \mathrm{MHz}): \delta 156.9$ (C, C-1), 156.5 (C, C-5), 137.1 (C, C-3), 113.8 (C, C-6), 110.3 (CH, C-2), 109.5 (CH, C-4), 83.5 (C, C-13), 74.3 (C, C-
7), 46.7 (CH, C-12), $37.2\left(\mathrm{CH}_{2}, \mathrm{C}-10\right), 35.2\left(\mathrm{CH}_{2}, \mathrm{C}-8\right), 29.6\left(\mathrm{CH}_{3}\right.$, $\left.\mathrm{C} 13-\mathrm{CH}_{3 \beta}\right), 29.0\left(\mathrm{CH}_{3}, \mathrm{C} 7-\mathrm{CH}_{3}\right), 27.9(\mathrm{CH}, \mathrm{C}-9), 23.6\left(\mathrm{CH}_{3}, \mathrm{C} 13-\right.$ $\left.\mathrm{CH}_{3 \alpha}\right), 22.0\left(\mathrm{CH}_{2}, \mathrm{C}-11\right), 21.6\left(\mathrm{CH}_{3}, \mathrm{C} 3-\mathrm{CH}_{3}\right)$.
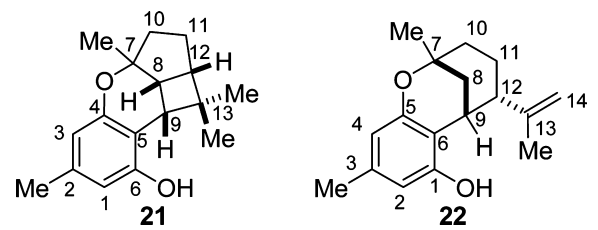

Ranhuadujuanine A (21) and Compound $22 .{ }^{24}$ Obtained from substrate $17(1.0 \mathrm{mmol}, 258 \mathrm{mg})$ as an inseparable mixture in $18 \%$ yield (0.18 mmol, $47 \mathrm{mg}, 21: 22 \mathrm{NMR}$ ratio 1:2). Yellow liquid. IR (film) $\left(\mathrm{cm}^{-1}\right): 3391,2930,1622,1587,1379$. Ranhuadujuanine A (21). ${ }^{1} \mathrm{H}$ NMR $\left(\mathrm{CDCl}_{3}, 300 \mathrm{MHz}\right): \delta 6.31\left(\mathrm{~s}, 1 \mathrm{H}, 4-\mathrm{H}^{*}\right), 6.17(\mathrm{~s}, 1 \mathrm{H}$, 2- $\left.\mathrm{H}^{*}\right), 4.44(\mathrm{~s}, 1 \mathrm{H}, \mathrm{OH}), 3.06$ (d, $\left.J=9.6 \mathrm{~Hz}, 1 \mathrm{H}, 9-\mathrm{H}\right), 2.57$ (dd, $J=$ $9.6 \mathrm{~Hz}, J=7.5 \mathrm{~Hz}, 1 \mathrm{H}, 8-\mathrm{H}), 2.39(\mathrm{bt}, J=7.6 \mathrm{~Hz}, 1 \mathrm{H}, 12-\mathrm{H}), 2.21(\mathrm{~s}$, $\left.3 \mathrm{H}, 3-\mathrm{CH}_{3}\right), 2.05-1.94(\mathrm{~m}, 1 \mathrm{H}, 10-\mathrm{H}), 1.70-1.57(\mathrm{~m}, 3 \mathrm{H}, 10-\mathrm{H}, 11-$ $\mathrm{H}, 11-\mathrm{H}), 1.37\left(\mathrm{~s}, 6 \mathrm{H}, 7-\mathrm{CH}_{3}, 13-\mathrm{CH}_{3 \beta}\right), 0.79\left(\mathrm{~s}, 3 \mathrm{H}, 13-\mathrm{CH}_{3 \alpha}\right) .{ }^{13} \mathrm{C}$ NMR $\left(\mathrm{CDCl}_{3}, 75 \mathrm{MHz}\right): \delta 154.0(2 \times \mathrm{C}, \mathrm{C}-1, \mathrm{C}-5), 137.3(\mathrm{C}, \mathrm{C}-3)$, 111.1 (CH, C-4**), 108.3 (C, C-6), 107.7 (CH, C-2**), 83.1 (C, C7), $46.2(\mathrm{CH}, \mathrm{C}-12), 38.8(\mathrm{C}, \mathrm{C}-13), 37.8\left(\mathrm{CH}_{2}, \mathrm{C}-10\right), 37.6(\mathrm{CH}, \mathrm{C}-$ 8), $35.8(\mathrm{CH}, \mathrm{C}-9), 33.8\left(\mathrm{CH}_{3}, \mathrm{C} 13-\mathrm{CH}_{3 \beta}\right), 27.5\left(\mathrm{CH}_{3}, \mathrm{C} 7-\mathrm{CH}_{3}\right)$, $25.5\left(\mathrm{CH}_{2}, \mathrm{C}-11\right), 21.1\left(\mathrm{CH}_{3}, \mathrm{C} 3-\mathrm{CH}_{3}\right), 17.8\left(\mathrm{CH}_{3}, \mathrm{C} 13-\mathrm{CH}_{3 \alpha}\right)$.

Compound 22. ${ }^{1} \mathrm{H}$ NMR $\left(\mathrm{CDCl}_{3}, 300 \mathrm{MHz}\right): \delta 6.27$ (bs, $1 \mathrm{H}, 4$ $\mathrm{H}), 6.18(\mathrm{bs}, 1 \mathrm{H}, 2-\mathrm{H}), 4.86(\mathrm{p}, J=1.5 \mathrm{~Hz}, 1 \mathrm{H}, 14-\mathrm{H}), 4.81(\mathrm{~s}, 1 \mathrm{H}$, $\mathrm{OH}), 4.65$ (bs, $1 \mathrm{H}, 14-\mathrm{H}), 3.30(\mathrm{q}, J=2.7 \mathrm{~Hz}, 1 \mathrm{H}, 9-\mathrm{H}), 2.41-2.30$ $(\mathrm{m}, 1 \mathrm{H}, 12-\mathrm{H}), 2.21\left(\mathrm{~s}, 3 \mathrm{H}, 3-\mathrm{CH}_{3}\right), 2.05-1.98(\mathrm{~m}, 1 \mathrm{H}, 10-\mathrm{H}), 1.92$ (dd, $J=12.9 \mathrm{~Hz}, J=2.9 \mathrm{~Hz}, 1 \mathrm{H}, 8-\mathrm{H}), 1.78\left(\mathrm{bs}, 3 \mathrm{H}, 13-\mathrm{CH}_{3}\right), 1.78$ $(\mathrm{dt}, J=12.7 \mathrm{~Hz}, J=3.3 \mathrm{~Hz}, 1 \mathrm{H}, 8-\mathrm{H}), 1.62-1.40(\mathrm{~m}, 3 \mathrm{H}, 10-\mathrm{H}, 11-\mathrm{H}$, $11-\mathrm{H}), 1.36\left(\mathrm{~s}, 3 \mathrm{H}, 7-\mathrm{CH}_{3}\right) \cdot{ }^{13} \mathrm{C} \mathrm{NMR}\left(\mathrm{CDCl}_{3}, 75 \mathrm{MHz}\right): \delta 157.0(\mathrm{C}$, C-5), 153.5 (C, C-1), 149.8 (C, C-13), 137.9 (C, C-3), $111.1\left(\mathrm{CH}_{2}, \mathrm{C}-\right.$ 14), $108.8(\mathrm{CH}, \mathrm{C}-4), 107.9(\mathrm{CH}, \mathrm{C}-2), 107.8$ (C, C-6), 73.7 (C, C7), $48.2(\mathrm{CH}, \mathrm{C}-12), 39.4\left(\mathrm{CH}_{2}, \mathrm{C}-10\right), 37.4\left(\mathrm{CH}_{2}, \mathrm{C}-8\right), 32.8(\mathrm{CH}$, $\mathrm{C}-9), 28.7\left(\mathrm{CH}_{3}, \mathrm{C} 7-\mathrm{CH}_{3}\right), 22.8\left(\mathrm{CH}_{2}, \mathrm{C}-11\right), 22.3\left(\mathrm{CH}_{3}, \mathrm{C} 13-\mathrm{CH}_{3}\right)$, $21.1\left(\mathrm{CH}_{3}, \mathrm{C} 3-\mathrm{CH}_{3}\right)$.

Iron Chloride Promoted Rearrangement of Substrate 6. To a solution of substrate $6(0.5 \mathrm{mmol})$ in $\mathrm{CH}_{2} \mathrm{Cl}_{2}(5.0 \mathrm{~mL}, 0.1 \mathrm{M})$ was added anhydrous $\mathrm{FeCl}_{3}(83.4 \mathrm{mg}, 0.5 \mathrm{mmol}, 1$ equiv), and the mixture was stirred at room temperature for $24 \mathrm{~h}$. The solvent was then evaporated under reduced pressure, and the residue was purified by flash column chromatography on silica gel (eluting with hexanes/ethyl acetate) to afford compounds 9 in $70 \%$ yield $(0.35 \mathrm{mmol}, 96 \mathrm{mg}$, cis/ trans ratio $=1: 0.14$ to $1: 0.2$ ).

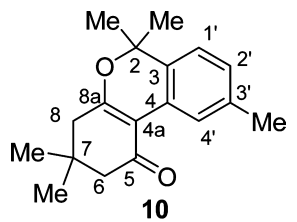

Chloranil Dehydrogenation of Compounds 9 for the Synthesis of 10. To a solution of substrates $9(21 \mathrm{mg}, 0.077$ $\mathrm{mmol}$, cis/trans ratio $=1: 1.2)$ in anhydrous toluene $(1.5 \mathrm{~mL}, 0.05 \mathrm{M})$ was added chloranil ( $18.8 \mathrm{mg}, 0.077 \mathrm{mmol}, 1$ equiv). The mixture was heated at reflux for $12 \mathrm{~h}$. The solvent was then evaporated under reduced pressure, and the residue was purified by flash column chromatography on silica gel (eluting with hexanes/ethyl acetate) to afford compound 10 in $35 \%$ yield $(7.2 \mathrm{mg}, 0.026 \mathrm{mmol})$ and unreacted cis-9 in approximately $45 \%$ yield $(9.5 \mathrm{mg}, 0.035 \mathrm{mmol})$. When a low trans-isomer content mixture of compounds 9 was employed as substrate $(60 \mathrm{mg}, 0.22 \mathrm{mmol}$, cis/trans ratio $=1: 0.2)$, compound 10 was obtained in approximately $15 \%$ yield ( $9 \mathrm{mg}, 0.033$ $\mathrm{mmol})$.

Compound 10. Colorless liquid. IR (film) $\left(\mathrm{cm}^{-1}\right)$ : 2959, 2916, $1655,1597,1377,1047 .{ }^{1} \mathrm{H}$ NMR $\left(\mathrm{CDCl}_{3}, 300 \mathrm{MHz}\right): \delta 8.34(\mathrm{bs}, 1 \mathrm{H}$, $\left.4^{\prime}-\mathrm{H}\right), 7.03\left(\mathrm{dd}, J=8.0 \mathrm{~Hz}, J=1.0 \mathrm{~Hz}, 1 \mathrm{H}, 2^{\prime}-\mathrm{H}\right), 6.99(\mathrm{~d}, J=7.8 \mathrm{~Hz}$, $\left.1 \mathrm{H}, 1^{\prime}-\mathrm{H}\right), 2.40(\mathrm{~s}, 2 \mathrm{H}, 6-\mathrm{H}), 2.38(\mathrm{~s}, 2 \mathrm{H}, 8-\mathrm{H}), 2.35\left(\mathrm{~s}, 3 \mathrm{H}, 3^{\prime}-\mathrm{CH}_{3}\right)$, $1.61\left(\mathrm{~s}, 6 \mathrm{H}, 2-\mathrm{CH}_{3}, 2-\mathrm{CH}_{3}\right), 1.10\left(\mathrm{~s}, 6 \mathrm{H}, 7-\mathrm{CH}_{3}, 7-\mathrm{CH}_{3}\right) .{ }^{13} \mathrm{C} \mathrm{NMR}$ $\left(\mathrm{CDCl}_{3}, 75 \mathrm{MHz}\right): \delta 196.2$ (C, C-5), 170.5 (C, C-8a), 137.3 (C, C- 
3'), 132.4 (C, C-3), 127.5 (CH, C-2'), 125.9 (C, C-4), 125.3 (CH, C$\left.4^{\prime}\right), 121.8\left(\mathrm{CH}, \mathrm{C}-1^{\prime}\right), 110.4(\mathrm{C}, \mathrm{C}-4 \mathrm{a}), 80.5(\mathrm{C}, \mathrm{C}-2), 52.3\left(\mathrm{CH}_{2}, \mathrm{C}-\right.$ 6), $43.5\left(\mathrm{CH}_{2}, \mathrm{C}-8\right), 31.4(\mathrm{C}, \mathrm{C}-7), 28.1\left(2 \times \mathrm{CH}_{3}, \mathrm{C} 7-\mathrm{CH}_{3}, \mathrm{C} 7-\right.$ $\left.\mathrm{CH}_{3}\right), 27.5\left(2 \times \mathrm{CH}_{3}, \mathrm{C} 2-\mathrm{CH}_{3}, \mathrm{C} 2-\mathrm{CH}_{3}\right), 21.3\left(\mathrm{CH}_{3}, \mathrm{C}^{\prime}-\mathrm{CH}_{3}\right)$. HRMS (ESI) $m / z$ calcd for $\mathrm{C}_{18} \mathrm{H}_{23} \mathrm{O}_{2}[\mathrm{M}+\mathrm{H}]^{+} 271.1693$, found 271.1691.

Synthesis of Benzosimuline (2). ${ }^{3 \mathrm{~b}}$ To a mixture of substrates 14 $\left(75.0 \mathrm{mg}, 0.24 \mathrm{mmol}\right.$, cis/trans ratio = 1:0.6), $\mathrm{MgSO}_{4}(25 \mathrm{mg})$, AMS ( $14.9 \mathrm{mg}, 0.048 \mathrm{mmol}, 0.2$ equiv), $p \mathrm{TsOH}$ monohydrate $(11.4 \mathrm{mg}$, $0.06 \mathrm{mmol}, 0.25$ equiv $)$, and $\mathrm{Pd}(\mathrm{OAc})_{2}(2.7 \mathrm{mg}, 0.012 \mathrm{mmol}, 0.05$ equiv) was added anhydrous 1,2-dichlorobenzene $(1 \mathrm{~mL}, 0.24 \mathrm{M})$. The reaction flask was purged with oxygen for $10 \mathrm{~min}$ using a needle inserted into the solvent. The solution was stirred while purging. An $\mathrm{O}_{2}$ balloon was then attached to the flask, this time with the needle above the solvent level, and the mixture was heated to $110^{\circ} \mathrm{C}$. After 2 $\mathrm{h}$, the mixture was diluted with hexanes and placed directly on a silica column which was run using hexanes/ethyl acetate as eluants to afford natural product 2 in $34 \%$ yield $(25 \mathrm{mg}, 0.08 \mathrm{mmol}$ ) along with unreacted cis-14 in $12 \%$ yield $(9 \mathrm{mg}, 0.03 \mathrm{mmol})$.

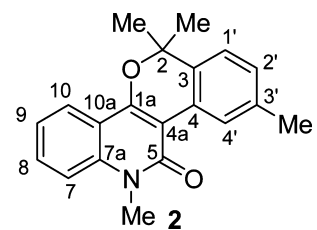

Compound 2. Colorless solid. Mp 139.7-140.9 ${ }^{\circ} \mathrm{C}$. IR ( $\mathrm{KBr}$ ) $\left(\mathrm{cm}^{-1}\right): 3082,2988,2964,2922,1638,1383,1115,1088 .{ }^{1} \mathrm{H}$ NMR $\left(\mathrm{CDCl}_{3}, 300 \mathrm{MHz}\right): \delta 8.80\left(\mathrm{~s}, 1 \mathrm{H}, 4^{\prime}-\mathrm{H}\right), 8.08(\mathrm{dd}, J=8.0 \mathrm{~Hz}, J=1.5$ $\mathrm{Hz}, 1 \mathrm{H}, 10-\mathrm{H}), 7.58(\mathrm{ddd}, J=8.6 \mathrm{~Hz}, J=7.2 \mathrm{~Hz}, J=1.6 \mathrm{~Hz}, 1 \mathrm{H}, 8-\mathrm{H})$, $7.36(\mathrm{bd}, J=8.4 \mathrm{~Hz}, 1 \mathrm{H}, 7-\mathrm{H}), 7.24(\mathrm{ddd}, J=8.1 \mathrm{~Hz}, J=7.3 \mathrm{~Hz}, J=$ $0.9 \mathrm{~Hz}, 1 \mathrm{H}, 9-\mathrm{H}), 7.15-7.08$ (m, 2H, $\left.1^{\prime}-\mathrm{H}, 2^{\prime}-\mathrm{H}\right), 3.78$ (bs, 3H, N$\left.\mathrm{CH}_{3}\right), 2.41\left(\mathrm{~s}, 3 \mathrm{H}, 3^{\prime}-\mathrm{CH}_{3}\right), 1.72\left(\mathrm{~s}, 6 \mathrm{H}, 2-\mathrm{CH}_{3}, 2-\mathrm{CH}_{3}\right) .{ }^{13} \mathrm{C} \mathrm{NMR}$ $\left(\mathrm{CDCl}_{3}, 75 \mathrm{MHz}\right): \delta 161.3$ (C, C-5), 155.8 (C, C-1a), 139.1 (C, C7a), 137.3 (C, C-3'), 134.2 (C, C-3), 131.0 (CH, C-8), 128.4 (CH, C$\left.2^{\prime}\right), 126.7$ (C, C-4), 126.1 (CH, C-4'), $123.5(\mathrm{CH}, \mathrm{C}-10), 121.9(\mathrm{CH}$, C-1'), $121.6(\mathrm{CH}, \mathrm{C}-9), 116.3$ (C, C-10a), $113.7(\mathrm{CH}, \mathrm{C}-7), 106.6(\mathrm{C}$, $\mathrm{C}-4 \mathrm{a}), 79.7(\mathrm{C}, \mathrm{C}-2), 29.2\left(\mathrm{CH}_{3}, \mathrm{~N}-\mathrm{CH}_{3}\right), 27.3\left(2 \times \mathrm{CH}_{3}, \mathrm{C} 2-\mathrm{CH}_{3}\right.$, $\left.\mathrm{C} 2-\mathrm{CH}_{3}\right), 21.4\left(\mathrm{CH}_{3}, \mathrm{C}^{\prime}-\mathrm{CH}_{3}\right)$. HRMS (ESI) $\mathrm{m} / z$ calcd for $\mathrm{C}_{20} \mathrm{H}_{19} \mathrm{NO}_{2} \mathrm{Na}[\mathrm{M}+\mathrm{Na}]^{+}$328.1308, found 328.1309.

\section{ASSOCIATED CONTENT}

\section{S Supporting Information}

The Supporting Information is available free of charge on the ACS Publications website at DOI: 10.1021/acs.joc.6b01278.

General experimental methods, full screening data, and copies of ${ }^{1} \mathrm{H},{ }^{13} \mathrm{C}$, and $2 \mathrm{D}$ NMR spectra for all compounds (PDF) (PDF)

\section{AUTHOR INFORMATION}

\section{Corresponding Authors}

*E-mail: riveira@iquir-conicet.gov.ar.

*E-mail: mischne@iquir-conicet.gov.ar.

\section{Notes}

The authors declare no competing financial interest.

\section{ACKNOWLEDGMENTS}

We thank Universidad Nacional de Rosario (BIO426), Fundación Josefina Prats, Consejo Nacional de Investigaciones Cientificas y Técnicas (CONICET, PIP 2012-2014 0448), and Agencia Nacional de Promoción Científica y Tecnológica (ANPCyT, BID-PICT-0408) for financial support. M.J.R. thanks CONICET for fellowships.

\section{REFERENCES}

(1) (a) Yang, S.-H.; Liu, Y.-Q.; Wang, J.-F.; Wang, Y.-H.; Pan, W.-W.; Sheng, W.-B.; Cai, R.; Zhou, X.-J. J. Antibiot. 2015, 68, 289-292. (b) Mansoor, T. A.; Borralho, P. M.; Luo, X.; Mulhovo, S.; Rodrigues, C. M. P.; Ferreira, M.-J. U. J. Nat. Prod. 2014, 77, 1825-1830. (c) Ahsan, M.; Haque, M. R.; Hossain, M. B.; Islam, S. N.; Gray, A. I.; Hasan, C. M. Phytochemistry 2014, 103, 8-12. (d) da Silva, M. F. G. F.; Fernandes, J. B.; Forim, M. R.; Vieira, P. C.; de Sá, I. C. G. In Natural Products; Ramawat, K. G., Mérillon, J. M., Eds.; SpringerVerlag: Berlin Heidelberg, 2013; pp 715-859. (e) Jangir, R.; Argade, N. P. RSC Adv. 2012, 2, 7087-7090. (f) Clement, B.; Weide, M.; Wolschendorf, U.; Kock, I. Angew. Chem., Int. Ed. 2005, 44, 635-638. (g) McLaughlin, M. J.; Hsung, R. P. J. Org. Chem. 2001, 66, 10491053.

(2) (a) Chen, I.-S.; Wu, S.-J.; Tsai, I.-L. J. Nat. Prod. 1994, 57, 12061211. (b) Wu, S.-J.; Chen, I.-S. Phytochemistry 1993, 34, 1659-1661.

(3) (a) Michael, J. P. Nat. Prod. Rep. 1999, 16, 697-709. (b) Chen, I. S.; Tsai, I.-W.; Teng, C.-M.; Chen, J.-J.; Chang, Y.-L.; Ko, F.-N.; Lu, M. C.; Pezzuto, J. M. Phytochemistry 1997, 46, 525-529.

(4) For a recent review on natural and synthetic chromenes, see: (a) Pratap, R.; Ram, V. J. Chem. Rev. 2014, 114, 10476-10526. For selected examples on the regioselective synthesis of some chromene derivatives, see: (b) Yadav, P.; Singh, S.; Sahu, S. N.; Hussain, F.; Pratap, R. Org. Biomol. Chem. 2014, 12, 2228-2234. (c) Pratap, R.; Kumar, R.; Maulik, P. R.; Ram, V. J. Tetrahedron Lett. 2007, 48, 33113314.

(5) (a) Taura, F.; Morimoto, S.; Shoyama, Y. J. Biol. Chem. 1996, 271, 17411-17416. (b) Razdan, R. K. In The Total Synthesis of Natural Products, ApSimon, J., Ed.; John Wiley \& Sons, Inc.: Hoboken, NJ, 1981; Vol. 4, pp 186-262. (c) Mechoulam, R.; McCallum, N. K.; Burstein, S. Chem. Rev. 1976, 76, 75-112. (d) Mechoulam, R. Science 1970, 168, 1159-1165.

(6) Grundon, M. F. In The Alkaloids, Arnold, B., Ed.; Academic Press: London, 1988; Vol. 32, pp 341-439.

(7) (a) Riveira, M. J.; Mischne, M. P. Synth. Commun. 2013, 43, 208220. (b) Wang, X.; Lee, Y. R. Synthesis 2007, 2007, 3044-3050. (c) Hsung, R. P.; Kurdyumov, A. V.; Sydorenko, N. Eur. J. Org. Chem. 2005, 2005, 23-44 and references cited therein.

(8) Tietze, L. F.; Kettschau, G. Top. Curr. Chem. 1997, 189, 1-120. (9) (a) Malerich, J. P.; Maimone, T. J.; Elliott, G. I.; Trauner, D. J Am. Chem. Soc. 2005, 127, 6276-6283. (b) Tapia, R. A.; Navarro, O.; Alegría, L.; Valderrama, J. A. Heterocycl. Commun. 1998, 4, 151-154.

(10) (a) Riveira, M. J.; Quiroga, G. N.; Mata, E. G.; Gandon, V.; Mischne, M. P. J. Org. Chem. 2015, 80, 6515-6519. (b) Li, H.; Tang, Y.; Hsung, R. P. Tetrahedron Lett. 2012, 53, 6138-6143. (c) Shen, H. C.; Wang, J.; Cole, K. P.; McLaughlin, M. J.; Morgan, C. D.; Douglas, C. J.; Hsung, R. P.; Coverdale, H. A.; Gerasyuto, A. I.; Hahn, J. M.; Liu, J.; Sklenicka, H. M.; Wei, L.-L.; Zehnder, L. R.; Zificsak, C. A. J. Org. Chem. 2003, 68, 1729-1735.

(11) (a) Garcia, A.; Borchardt, D.; Chang, C.-E. A.; Marsella, M. J. J. Am. Chem. Soc. 2009, 131, 16640-16641. For some other notable pericyclic cascades, see: (b) Richmond, E.; Duguet, N.; Slawin, A. M. Z.; Lébl, T.; Smith, A. D. Org. Lett. 2012, 14, 2762-2765. (c) ÇelebiÖlçüm, N.; Lam, Y.; Richmond, E.; Ling, K. B.; Smith, A. D.; Houk, K. N. Angew. Chem., Int. Ed. 2011, 50, 11478-11482. (d) Steinhardt, S. E.; Vanderwal, C. D. J. Am. Chem. Soc. 2009, 131, 7546-7547. (e) Miller, A. K.; Trauner, D. Angew. Chem., Int. Ed. 2005, 44, 46024606. (f) Li, C.; Lobkovsky, E.; Porco, J. A., Jr. J. Am. Chem. Soc. 2000, 122, 10484-10485. For general reviews on domino or cascade processes, see: (g) Padwa, A.; Bur, S. K. Tetrahedron 2007, 63, 53415378. (h) Nicolaou, K. C.; Edmonds, D. J.; Bulger, P. G. Angew. Chem., Int. Ed. 2006, 45, 7134-7186. (i) Tietze, L. F. Chem. Rev. 1996, 96, $115-136$.

(12) Irradiation has been shown to promote ring opening of chromene derivatives, for example, see: (a) Padwa, A.; Au, A.; Lee, G. A.; Owens, W. J. Org. Chem. 1975, 40, 1142-1149. In our case, a photochemical approach was originally avoided since prenyl-side chain bearing chromenes have been shown to undergo intramolecular $[2+$ 2] cycloaddition to afford cyclobutanes, for example, see: (b) Kurdyu- 
mov, A. V.; Hsung, R. P.; Ihlen, K.; Wang, J. Org. Lett. 2003, 5, 39353938. For some unfruitful photochemical attempts to produce cycloadducts 9, see Table S1 in the Supporting Information.

(13) Tietze, L. F.; Geissler, H.; Fennen, J.; Brumby, T.; Brand, S.; Schulz, G. J. Org. Chem. 1994, 59, 182-191.

(14) Lo, J. C.; Yabe, Y.; Baran, P. S. J. Am. Chem. Soc. 2014, 136, 1304-1307.

(15) For comparison purposes, related natural product cannabidiol (CBD) cyclizes to THC upon acid treatment: (a) Gaoni, Y.; Mechoulam, R. J. Am. Chem. Soc. 1964, 86, 1646-1647. For other related examples, see: (b) Crombie, L.; Crombie, W. M. L.; Tuchinda, P. J. Chem. Soc., Perkin Trans. 1 1988, 1255-1262.

(16) Turk, R. F.; Manno, J. E.; Jain, N. C.; Forney, R. B. J. Pharm. Pharmacol. 1971, 23, 190-195.

(17) Mechoulam, R.; Yagnitinsky, B.; Gaoni, Y. J. Am. Chem. Soc. 1968, 90, 2418-2420.

(18) (a) Adams, R.; Pease, D. C.; Cain, C. K.; Clark, J. H. J. Am. Chem. Soc. 1940, 62, 2402-2405. (b) Ghosh, R.; Todd, A. R; Wilkinson, S. J. Chem. Soc. 1940, 1393-1396.

(19) Kirsch, S. F.; Wegener, M. In Comprehensive Organic Synthesis, 2nd ed.; Molander, G. A., Knochel, P., Eds.; Elsevier: Oxford, 2014; Vol. 7, pp 1-25.

(20) (a) Iosub, A. V.; Stahl, S. S. J. Am. Chem. Soc. 2015, 137, 34543457. (b) Kandukuri, S. R.; Oestreich, M. J. Org. Chem. 2012, 77, 8750-8755. (c) Horrillo-Martínez, P.; Virolleaud, M.-A.; Jaekel, C. ChemCatChem 2010, 2, 175-181. (d) Jiang, M.; Wei, Y.; Shi, M. Eur. J. Org. Chem. 2010, 2010, 3307-3311.

(21) Vree, T. B.; Breimer, D. D.; Van Ginneken, C. A. M.; Van Rossum, J. M. J. Pharm. Pharmacol. 1972, 24, 7-12.

(22) Lee, Y. R.; Choi, J. H.; Yoon, S. H. Tetrahedron Lett. 2005, 46, $7539-7543$.

(23) In this case, our solvent-free condensation protocol failed to promote any reaction of the resorcinol substrate.

(24) (a) Li, X.; Lee, Y. R. Org. Biomol. Chem. 2014, 12, 1250-1257. (b) Kane, V. V.; Martin, A. R.; Peters, J. A. J. Org. Chem. 1984, 49, 1793-1796.

(25) (a) Kurdyumov, A. V.; Hsung, R. P. J. Am. Chem. Soc. 2006, 128, 6272-6273. (b) Gassman, P. G.; Chavan, S. P.; Fertel, L. B. Tetrahedron Lett. 1990, 31, 6489-6492.

(26) (a) Van de Water, R. W.; Pettus, T. R. R. Tetrahedron 2002, 58, 5367-5405. (b) Marino, J. P.; Dax, S. L. J. Org. Chem. 1984, 49, 3671-3672. 